\title{
The impact of Maternal and Child Health and Nutrition Improvement Project on maternal and child health outcomes and service utilization in Ghana: An interrupted time series analysis
}

\author{
Arif Mohammed \\ University of Ghana School of Public Health \\ Duah Dwomoh \\ University of Ghana School of Public Health \\ Justice Nonvignon ( $\square$ jnonvignon@ug.edu.gh ) \\ University of Ghana School of Public Health
}

\section{Research article}

Keywords: Maternal and Child Health and Nutrition Improvement Project, Impact, Performance-based financing, Maternal and Child Health, Interrupted time series analysis, ANC visits, Maternal Mortality Rate, Neonatal Mortality Rate, Skilled delivery

Posted Date: May 7th, 2020

DOI: https://doi.org/10.21203/rs.3.rs-25287/v1

License: (c) (1) This work is licensed under a Creative Commons Attribution 4.0 International License. Read Full License 


\section{Abstract \\ Background}

Improving maternal and child health outcomes $(\mathrm{MCH})$ continues to be a major public health concern to governments in sub-Saharan Africa and the international development community. The Maternal and Child Health and Nutrition Improvement Project (MCHNP) was a nationwide project that sought to improve the utilization of maternal and child health services in Ghana through financial incentive packages. The objective of this study was to determine the differential impact of MCHNP on maternal and child health outcomes.

\section{Methods}

A retrospective longitudinal pre-test post-test study design was employed. The study used monthly data from the District Health Information Management System between January 2014 to December 2018. Interrupted time series analysis was applied to estimate the impact of MCHNP on $\mathrm{MCH}$ for each region of the country.

\section{Results}

Neonatal mortality rate increased significantly in the Western,Upper East and Upper West regions with impact estimates of 0.144 ( $95 \% \mathrm{Cl}$ : $0.100,0.188 ; p$-value < 0.001), 0.124 (95\% Cl: 0.076, 0.172; p-value< 0.001$)$ and 0.082 (95\% Cl: 0.048, 0.117; p-value< 0.001$)$ respectively. The proportion of women who had four ANC visits decreased significantly in the Volta region with an impact of -0.011 ( $95 \% \mathrm{Cl}:-0.020$, -0.002; p-value < 0.01), but increased in the Central, Western, Eastern, Upper East, and Upper West with estimated impacts of 0.004 ( $95 \% \mathrm{Cl}$ : $-0.003,0.011$ ), 0.003 (95\% Cl: -0.003, 0.008), 0.002 (95\% Cl: -0.004, 0.008), 0.011 (95\% Cl: -0.004, 0.026) and 0.007 (95\% Cl: -0.012, 0.026) respectively though none of the increase was statistically significant. Skilled deliveries reduced in the Greater Accra, Volta, Ashanti and Brong-Ahafo regions with statistically insignificant estimated impacts of 0.006 ( $95 \% \mathrm{Cl}:-0.019,0.007), 0.005$ ( $95 \% \mathrm{Cl}:-0.015,0.006), 0.005$ (95\% Cl: $-0.011,0.002)$ and $0.004(95 \% \mathrm{Cl}:-0.010,0.002)$ respectively. Maternal mortality rate declined in Greater Accra by; 0.001 (95\% Cl: $-0.018,0.017$ ), Volta; -0.010 (95\% Cl: -0.035, 0.016), Western; -0.009 (95\% Cl: -0.031, 0.013), Brong-Ahafo; -0.018 (95\% Cl: -0.052, 0.017), Eastern; -0.025 (95\% Cl: $-0.064,0.013)$ and Northern - 0.024 (95\% Cl: $-0.060,0.011)$ regions.

\section{Conclusion}

The nationwide implementation of MCHNP produced mixed results as some regions recorded positive (significant) impacts whereas others had no significant impacts on the outcome variables as expected following the implementation of the intervention, therefore the need for further studies to understand why the intervention failed to produce positive impacts in some regions.

\section{Background}

Maternal and child health continue to receive major attention on the global health lanscape as evidenced in major global goals such as the sustainable development goals (SDGs) [1]. A joint report by the World Health Organization and UNICEF in 2014 revealed an improvement in maternal health services utilization, with about $86 \%$ of pregnant women accessing prenatal care globally $[2,3]$. Clavagnier (2014) indicated that $50 \%$ of women in sub-Saharan Africa (SSA) were able to receive the WHO recommended four ANC attendance [4] and $58 \%$ of children were delivered by a skilled birth attendant [5].

Poor socioeconomic status, high user fees, inaccessibility to essential healthcare services, poorly motivated providers and reduced service quality in many healthcare setting have been identified as causes of low utilization of maternal health services $[6,7,8]$. These factors have led to the death of over half a million women during the prenatal, delivery or the postnatal stages[6].

The Maternal and Child Health and Nutrition Improvement Project (MCHNP) is a performance-based financing (PBF) initiative that sought to improve maternal and child health service utilization for positive outcomes [9]. Performance-based financing refers to the allocation of resources to a beneficiary, conditional on the recipient taking a measurable action or achieving a predetermined performance target [10]. MCHNP as a PBF intiative aims to make essential healthcare services accessible to, and deliver available services to the doorsteps of the population especially those in hard to reach areas where access to healthcare is a major bottleneck $[11,12]$. 
The introduction of MCHNP in Ghana was based on wider disparities that existed in the country which affected use of maternal and child health services especially in remote communities. To reiterate, the Ghana Health Service (GHS) and Demographic Heath Survey (DHS) indicated in 2015 that only $41 \%$ of pregnant women in rural Ghana received skilled deliveries [13], clearly indicating low service utilization, although the country saw an improvement over the last decade in its maternal and child health outcomes, where it recorded a 380 maternal deaths per 100,000 live births and 60 child deaths in 1,000 live births. [14].

PBF initiatives in recent times have received several critiques following the Cochrane collaboration systematic review on PBF in 2012, which indicates that PBF in low and middle-income countries are based on too weak evidence and very little experience for a large scale implementation, hence the tendency to weaken the existing health system $[15,16]$. The objective of this study, therefore, was to determine the differential impact of MCHNP on maternal and child health service utilization and outcomes in Ghana.

\section{Methods}

\section{Study design}

The study employed a retrospective pre and post-test study design, which is a longitudinal study design. Monthly data on outcome variables were collected over January 2014 to December 2018. The adoption of the interrupted time series design was as a result of the lack of a control group, given that the intervention was implemented nationwide.

\section{Study setting}

The study was conducted in all the ten (10) administrative regions of Ghana as of 2018 namely the Ashanti, Brong-Ahafo, Central, Eastern, Greater Accra, Northern, Upper East, Upper West, Volta and Western regions.

\section{Study population}

The study populations were pregnant women, post-partum women who accessed services obstetric services as well as those who died in the course of receiving these services, and babies who died within the first 28 days of life under the review periods in all 10 regions of Ghana.

\section{Data collection}

Data for the study were obtained from the national health database District Health Information Management System II (DHIMS2). Data on the outcome variables were extracted from the various datasets in the form of a query for the period January 2014 to December 2018 and for the ten (10) administrative regions in the country as of 2018 using the pivot table application in the DHIMS2 database. Results from the queries were downloaded into an excel template for cleaning and management.

\section{Study variables}

\begin{tabular}{|c|c|c|c|}
\hline Variable & $\begin{array}{l}\text { Type of } \\
\text { variable }\end{array}$ & Description & Measurement \\
\hline Antenatal four visits & $\begin{array}{l}\text { Outcome } \\
\text { variable }\end{array}$ & $\begin{array}{l}\text { Number of pregnant women that visited the facility for a minimum } \\
\text { of four times for prenatal services }\end{array}$ & Count \\
\hline Skillled Delivery & $\begin{array}{l}\text { Outcome } \\
\text { variable }\end{array}$ & $\begin{array}{l}\text { Number of pregnant women who were delivered by qualified } \\
\text { professionals }\end{array}$ & Count \\
\hline Maternal Mortality & $\begin{array}{l}\text { Outcome } \\
\text { variable }\end{array}$ & $\begin{array}{l}\text { Women who died while pregnant or in their postpartm peroid ( } 42 \\
\text { days), or during the termination of a pregnancy, or its } \\
\text { management }\end{array}$ & Count \\
\hline Neonatal mortality & Outcome & Death of infants between the ages of zero to 28 days of life & Count \\
\hline $\begin{array}{l}\text { Maternal and Child Health and } \\
\text { Nutrition improvement Project }\end{array}$ & Explanatory & $\begin{array}{l}\text { The Performance-based financing Intervention been evaluated and } \\
\text { when it was started }\end{array}$ & $\begin{array}{l}\text { Binary (Yes, } \\
\text { No) }\end{array}$ \\
\hline
\end{tabular}

\section{Data management}

Data extracted were validated to identify any inconsistencies, incompleteness, and inaccuracies. Inconsistent data were compared with data in the other datasets in the system as a data element may appear more than once in the system to make room for checks and balances during data entry or transcriptions. This was done to enhance reliability in the data as well as producing a credible study result. 
The data extracted were saved unto a hard disk drive and were kept in a password-protected file to prevent unauthorized personnel's from accessing and making changes to the data which might affect consistency in the study results. Again a copy of the data was drafted into a cloud storage space to enable access to the data in case of any catastrophe.

\section{Statistical analysis}

Background information on maternal and child health service coverage, utilization and outcomes were summarized and further segregated into pre-intervention summary (January 2014-December, 2015) and post-intervention summary (January 2016 - December 2018).

Normality tests were carried out prior to the impact analysis to indicate whether or not the data are normally distributed using histogram super-imposed with a normal distribution curve and the Shapiro-Francia Test of Gaussian distribution. The mean comparison of the outcome measures pre and post-intervention periods was estimated using unequal Welch t-test for each of the dependent variables.

The evaluated intervention is said to target population-level health outcomes because the intervention was implemented at the population level with data collected at regular time intervals (monthly) over a specified period of time indicating clearly the pre and post-intervention periods with outcomes analyzed at the regional level to achieve the study objectives. An interrupted time-series analysis (ITSA) regression model was fixed to determine the differential impact of MCHNP on the observed health outcome variables using the underlying trends determined over the study period.

An interrupted time series analysis (ITSA) is applicable when taking into account the effect of an intervention at a population level and the available data are reported at an aggregated level and when several measurements on the outcome variables are obtainable pre and postintervention period as it offers a hypothetically high level of internal validity [17].

Time series data are said to encounter serial correlation or autocorrelation (due to similarity between observations at a different time) as well as heteroscedasticity (due to differences in variability among the various subpopulations) in the model's error term. Cumby-Huizinga test for autocorrelation was employed to overcome serial correlation and heteroscedasticity at specified lags.

\section{Estimation technique}

Interrupted time series analysis (ITSA) relies on ordinary least square (OLS) regression due to its flexibility in the context of ITSA and its ability to account for autocorrelated errors [18].

A single-group ITSA must be employed when the study has no comparison group that is, used only one group [18, 19], hence the adoption of the single-group ITSA with the regression equation presented as below;

$$
Y=\beta_{0}+\beta_{1} T+\beta_{2} X+\beta_{3} T X+\varepsilon
$$

Where; $\mathbf{Y}$ is the outcome variable of interest measured, $\mathbf{T}$ is the time periods specified for the study, $\mathbf{X}$ is a dummy variable representing the intervention (pre-intervention periods 0 , post-intervention 1), TX is an interaction term and represent the impact of the intervention and $\boldsymbol{\epsilon}$ been the error term.

In the case of a single-group study, $\boldsymbol{\beta}_{0}$ represents the intercept or starting level of the outcome variable. $\boldsymbol{\beta}_{\boldsymbol{1}}$ is the slope or trajectory of the outcome variable until the introduction of the intervention. $\boldsymbol{\beta}_{2}$ represents the change in the level of the outcome that occurs in the period immediately following the introduction of the intervention (compared with the counterfactual). $\boldsymbol{\beta}_{3}$ represents the difference between preintervention and post-intervention slopes of the outcome variables" [18].

Statistical analysis and presentations were conducted using Stata IC 15.0 (StataCorp, College Station, USA) and MS Excel and a p-value of 0.05 was considered statistically significant.

The statistical analysis was followed by a plausibility argument in discussing the variations if any in the performances of the measured indicators across all the ten (10) regions of Ghana.

\section{Results}




\section{Background characteristics of maternal and child health services and outcomes}

Table 1 presents background characteristics of the study population and intervention. Antenatal clinic (ANC) registrants increased by 47.5\% post-intervention and a 59.7\% increase in the number of pregnant women presenting for delivery. Deliveries conducted by TBAs post-intervention dwindled by $32.6 \%$ and also there was a reduction of $12.7 \%$ in stillbirths post-intervention. The maternal mortality rate decreased by $0.98 \%$ following the implementation of the intervention, but neonatal mortalities rate increased by $78.4 \%$ post-intervention. 
Table 1

Background characteristics of maternal and child health services and outcomes

\begin{tabular}{|c|c|c|c|c|}
\hline Characteristics & Pre-intervention (n) & Percentage (\%) & Post-intervention (n) & Percentage (\%) \\
\hline \multicolumn{5}{|l|}{ Antenatal } \\
\hline \multicolumn{5}{|c|}{ Age group of registrants } \\
\hline $10-14$ & 5,987 & 0.31 & 7,611 & 0.27 \\
\hline $15-19$ & 225,109 & 11.82 & 327,791 & 11.67 \\
\hline $20-24$ & 487,548 & 25.60 & 682,292 & 24.29 \\
\hline $25-29$ & 540,034 & 28.36 & 786,080 & 27.99 \\
\hline $30-34$ & 400,264 & 21.02 & 613,506 & 21.84 \\
\hline 35 and above & 245,401 & 12.89 & 391,428 & 13.94 \\
\hline \multicolumn{5}{|l|}{ Trimester } \\
\hline 1st trimester & 862,623 & 45.75 & $1,328,024$ & 47.55 \\
\hline 2nd trimester & 784,834 & 41.62 & $1,144,749$ & 40.99 \\
\hline 3rd trimester & 238,087 & 12.63 & 319,901 & 11.46 \\
\hline \multicolumn{5}{|l|}{ Parity } \\
\hline 0 & 525,081 & 27.69 & 775,416 & 27.72 \\
\hline $1-2$ & 751,488 & 39.63 & $1,126,637$ & 40.27 \\
\hline $3-4$ & 433,342 & 22.85 & 635,121 & 22.70 \\
\hline 5 and above & 186,290 & 9.82 & 260,532 & 9.31 \\
\hline \multicolumn{5}{|c|}{ Intermittent Preventive Treatment } \\
\hline IPT 1 & $1.171,653$ & 39.41 & $1,907,234$ & 34.33 \\
\hline IPT 2 & 920,356 & 30.96 & $1,599,566$ & 28.79 \\
\hline IPT 3 & 626,629 & 21.08 & $1,193,778$ & 21.49 \\
\hline IPT 4 & 188,553 & 6.34 & 606,141 & 10.91 \\
\hline IPT 5 & 65,801 & 2.21 & 248,724 & 4.48 \\
\hline \multicolumn{5}{|l|}{ Delivery } \\
\hline \multicolumn{5}{|c|}{ Age Groups at Delivery } \\
\hline $10-14$ & 3,387 & 0.27 & 4,899 & 0.25 \\
\hline $15-19$ & 140,902 & 11.33 & 223,142 & 11.23 \\
\hline $20-24$ & 305,370 & 24.55 & 461,535 & 23.23 \\
\hline $25-29$ & 354,105 & 28.47 & 561,651 & 28.27 \\
\hline $30-34$ & 270,404 & 21.74 & 446,691 & 22.49 \\
\hline 35 and above & 169,601 & 13.64 & 288,515 & 14.52 \\
\hline \multicolumn{5}{|l|}{ Type of Delivery } \\
\hline Normal & $1,056,691$ & 84.95 & 1635,241 & 82.77 \\
\hline \multicolumn{5}{|l|}{ LB: Live births } \\
\hline \multicolumn{5}{|c|}{ GHS: Ghana Health Service } \\
\hline CHAG: Christian & ociation of Ghana & & & \\
\hline
\end{tabular}




\begin{tabular}{|c|c|c|c|c|}
\hline Characteristics & Pre-intervention (n) & Percentage (\%) & Post-intervention (n) & Percentage (\%) \\
\hline Caesarean section & 187,252 & 15.05 & 340,380 & 17.23 \\
\hline \multicolumn{5}{|l|}{ Site of Delivery } \\
\hline GHS Facilities & 833,384 & 55.24 & $1,366,701$ & 59.18 \\
\hline CHAG & 241,331 & 16.00 & 373,227 & 16.16 \\
\hline Teaching Hospital & 48,146 & 3.19 & 81,502 & 3.53 \\
\hline Quasi Facilities & 31,162 & 2.07 & 42,385 & 1.84 \\
\hline Private Facilities & 201,891 & 13.38 & 288,025 & 12.47 \\
\hline TBA deliveries & 152,866 & 10.13 & 157,412 & 6.82 \\
\hline \multicolumn{5}{|l|}{ Outcome of Delivery } \\
\hline Live births & $1,306,441$ & 98.27 & $1,963,564$ & 98.49 \\
\hline Stillbirths & 23,012 & 1.73 & 30,197 & 1.51 \\
\hline \multicolumn{5}{|l|}{ Mortalities } \\
\hline \multicolumn{5}{|c|}{ Age Group of Maternal deaths } \\
\hline $10-14$ & 11 & 0.59 & 5 & 0.18 \\
\hline $15-19$ & 149 & 7.98 & 178 & 6.41 \\
\hline $20-24$ & 304 & 16.28 & 409 & 14.72 \\
\hline $25-29$ & 462 & 24.75 & 692 & 24.90 \\
\hline $30-34$ & 483 & 25.87 & 761 & 27.38 \\
\hline 35 and above & 458 & 24.53 & 734 & 26.41 \\
\hline Neonatal deaths & 4,823 & $3.7 / 1000 \mathrm{LB}$ & 13,101 & 6.6/1000LB \\
\hline \multicolumn{5}{|l|}{ LB: Live births } \\
\hline \multicolumn{5}{|c|}{ GHS: Ghana Health Service } \\
\hline CHAG: Christian $\mathrm{He}$ & sociation of Ghana & & & \\
\hline
\end{tabular}

\section{Nationwide comparison of pre and post-intervention means of the outcome variables using unequal Welch t-test}

The mean ANC four visit pre and post-intervention was estimated at 3.86 (95\% Cl: 3.69, 4.05; p-value = 0.42) and 3.97 (95\% Cl: 3.80, 4.14; $\mathrm{p}$-value $=0.42$ ) respectively as shown in Table 2 . There was a statistically significant difference in the mean skilled delivery with an estimated pre and post-intervention mean of $3.37(95 \% \mathrm{Cl}: 3.24,3.50$; p-value $<0.001)$ and $3.70(3.57,3.82 ; \mathrm{p}$-value $<0.001)$ respectively. Mean maternal mortality rate pre-intervention was estimated at 4.86 per 100,000 live births $(95 \% \mathrm{Cl}: 4.79,4.92 ; \mathrm{p}$-value $=0.54)$ and a postintervention mean of 4.83 per 100,000 live births $(95 \% \mathrm{Cl}$ : 4.78, 4.88; $\mathrm{p}$-value $=0.54)$. Pre and post-intervention neonatal mortality rate mean was estimated at 0.98 per 1,000 live births ( $95 \% \mathrm{Cl}: 0.86,1.10$; $p$-value < 0.001$)$ and 1.60 per 1,000 live births $(95 \%$ Cl: $1.52,1.68 ; p$ value $<0.001$ ) respectively. 
Table 2

Nationwide comparison of pre and post-intervention means of the outcome variables using unequal Welch t-test

\begin{tabular}{|c|c|c|c|c|}
\hline Variables & Mean & Standard Deviation & P-value & $95 \% \mathrm{Cl}$ \\
\hline \multicolumn{5}{|c|}{ Percentage of women who had 4 ANC visits } \\
\hline Pre-intervention & 3.86 & 1.45 & 0.42 & {$[3.69 ; 4.05]$} \\
\hline Post intervention & 3.97 & 1.64 & & {$[3.80,4.14]$} \\
\hline \multicolumn{5}{|c|}{ Percentage of women who had skilled delivery } \\
\hline Pre-intervention & 3.37 & 1.01 & $<0.001^{\star \star *}$ & {$[3.24,3.50]$} \\
\hline Post intervention & 3.70 & 1.22 & & {$[3.57,3.82]$} \\
\hline \multicolumn{5}{|c|}{ Maternal Mortality Rate (per 100,000 LBs) } \\
\hline Pre-intervention & 4.86 & 0.51 & 0.54 & {$[4.79,4.92]$} \\
\hline Post intervention & 4.83 & 0.50 & & {$[4.78,4.88]$} \\
\hline \multicolumn{5}{|c|}{ Neonatal Mortality Rate (per 1,000 LBs) } \\
\hline Pre-intervention & 0.98 & 0.93 & $<0.001 * * \star$ & {$[0.86,1.10]$} \\
\hline Post intervention & 1.60 & 0.76 & & {$[1.52,1.68]$} \\
\hline Significance level: & $\star \star * p<0$ & $01,{ }^{* *} p<0.01,{ }^{*} p<0.0$ & & \\
\hline
\end{tabular}

\section{T-test comparing Pre and Post-intervention means of outcome variables by regions}

Table 3 shows results of mean pre and post-intervention of all the outcome variables using unequal Welch t-test in the respective regions. 
Table 3

T-test comparing Pre and Post-intervention means of outcome variables by regions

\begin{tabular}{|c|c|c|c|c|c|c|c|c|}
\hline \multirow[t]{2}{*}{ Variables } & \multicolumn{2}{|l|}{ ANC4 visit } & \multicolumn{2}{|l|}{ Skilled Delivery } & \multicolumn{2}{|c|}{ Maternal Mortality rate } & \multicolumn{2}{|c|}{ Neonatal Mortality Rate } \\
\hline & $\begin{array}{l}\text { Pre- } \\
\text { intervention }\end{array}$ & $\begin{array}{l}\text { Post } \\
\text { intervention }\end{array}$ & $\begin{array}{l}\text { Pre- } \\
\text { intervention }\end{array}$ & $\begin{array}{l}\text { Post } \\
\text { intervention }\end{array}$ & $\begin{array}{l}\text { Pre- } \\
\text { intervention }\end{array}$ & $\begin{array}{l}\text { Post } \\
\text { intervention }\end{array}$ & $\begin{array}{l}\text { Pre- } \\
\text { intervention }\end{array}$ & $\begin{array}{l}\text { Post } \\
\text { intervention }\end{array}$ \\
\hline \multicolumn{9}{|l|}{ Central } \\
\hline $\begin{array}{l}\text { Mean } \\
{[95 \% \mathrm{Cl}]}\end{array}$ & $\begin{array}{l}4.05[3.89 \\
4.23]\end{array}$ & $\begin{array}{l}3.73[3.59, \\
3.86]\end{array}$ & $\begin{array}{l}1.27[1.22, \\
1.32]\end{array}$ & $\begin{array}{l}1.29[1.25, \\
1.33]\end{array}$ & $\begin{array}{l}4.57[4.31 \\
4.82]\end{array}$ & $\begin{array}{l}4.87[4.73 \\
5.01]\end{array}$ & $\begin{array}{l}0.35[-0.12, \\
0.82]\end{array}$ & $\begin{array}{l}1.37[1.17, \\
1.57]\end{array}$ \\
\hline P-value & $<0.01$ & & 0.55 & & 0.04 & & $<0.001$ & \\
\hline \multicolumn{9}{|c|}{ Greater Accra } \\
\hline $\begin{array}{l}\text { Mean } \\
{[95 \% \mathrm{Cl}]}\end{array}$ & $\begin{array}{l}5.80[5.44, \\
6.15]\end{array}$ & $\begin{array}{l}5.89[5.64, \\
6.13]\end{array}$ & $\begin{array}{l}1.58 \text { [1.49, } \\
1.66]\end{array}$ & $\begin{array}{l}1.55 \text { [1.50, } \\
1.59]\end{array}$ & $\begin{array}{l}5.18 \text { [5.06, } \\
5.30]\end{array}$ & $\begin{array}{l}5.11[5.00, \\
5.21]\end{array}$ & $\begin{array}{l}1.70[1.49, \\
1.92]\end{array}$ & $\begin{array}{l}2.07 \text { [1.93, } \\
2.20]\end{array}$ \\
\hline P-value & 0.67 & & 0.51 & & 0.37 & & $<0.01$ & \\
\hline \multicolumn{9}{|l|}{ Volta } \\
\hline $\begin{array}{l}\text { Mean } \\
{[95 \% \mathrm{Cl}]}\end{array}$ & $\begin{array}{l}2.94[2.74, \\
3.14]\end{array}$ & $\begin{array}{l}3.09 \text { [2.99, } \\
3.20]\end{array}$ & $\begin{array}{l}0.93[0.86, \\
1.01]\end{array}$ & $\begin{array}{l}1.09 \text { [1.05, } \\
1.13]\end{array}$ & $\begin{array}{l}4.98[4.83, \\
5.14]\end{array}$ & $\begin{array}{l}4.68 \text { [4.47, } \\
4.89]\end{array}$ & $\begin{array}{l}1.16[0.99, \\
1.32]\end{array}$ & $\begin{array}{l}1.63[1.51, \\
1.76]\end{array}$ \\
\hline P-value & 0.18 & & $<0.001$ & & 0.02 & & $<0.001$ & \\
\hline \multicolumn{9}{|l|}{ Western } \\
\hline $\begin{array}{l}\text { Mean } \\
{[95 \% \mathrm{Cl}]}\end{array}$ & $\begin{array}{l}3.31 \text { [3.16, } \\
3.45]\end{array}$ & $\begin{array}{l}3.13 \text { [3.03, } \\
3.22]\end{array}$ & $\begin{array}{l}1.00 \text { [0.96, } \\
1.03]\end{array}$ & $\begin{array}{l}1.10[1.06, \\
1.14]\end{array}$ & $\begin{array}{l}4.82 \text { [4.62, } \\
5.02]\end{array}$ & $\begin{array}{l}4.82[4.70 \\
4.95]\end{array}$ & $\begin{array}{l}0.99[0.59 \\
1.40]\end{array}$ & $\begin{array}{l}1.31[1.07, \\
1.54]\end{array}$ \\
\hline P-value & 0.04 & & $<0.001$ & & 0.97 & & 0.18 & \\
\hline \multicolumn{9}{|l|}{ Ashanti } \\
\hline $\begin{array}{l}\text { Mean } \\
{[95 \% \mathrm{Cl}]}\end{array}$ & $\begin{array}{l}4.66[4.40, \\
4.91]\end{array}$ & $\begin{array}{l}3.66 \text { [3.51, } \\
3.81]\end{array}$ & $1.33[1.30,1.37]$ & $\begin{array}{l}1.30[ \\
1.27 \\
1.33]\end{array}$ & $\begin{array}{l}4.70[4.48, \\
4.92]\end{array}$ & $\begin{array}{l}4.88[4.71 \\
5.05]\end{array}$ & $\begin{array}{l}0.17[-0.29 \\
0.64]\end{array}$ & $\begin{array}{l}1.82[1.45, \\
2.20]\end{array}$ \\
\hline P-value & $<0.001$ & & 0.20 & & 0.21 & & $<0.001$ & \\
\hline \multicolumn{9}{|c|}{ Brong-Ahafo } \\
\hline $\begin{array}{l}\text { Mean } \\
{[95 \% \mathrm{Cl}]}\end{array}$ & $\begin{array}{l}2.57 \text { [2.39, } \\
2.76]\end{array}$ & $\begin{array}{l}2.97 \text { [2.83, } \\
3.11]\end{array}$ & $0.88[0.85,0.91]$ & $\begin{array}{l}0.98 \\
{[0.94} \\
1.02]\end{array}$ & $\begin{array}{l}4.82[4.65, \\
5.00]\end{array}$ & $\begin{array}{l}4.55[4.39 \\
4.71]\end{array}$ & $\begin{array}{l}1.68[1.54, \\
1.81]\end{array}$ & $\begin{array}{l}1.97[1.89 \\
2.05]\end{array}$ \\
\hline P-value & 0.001 & & $<0.001$ & & 0.02 & & $<0.001$ & \\
\hline \multicolumn{9}{|l|}{ Eastern } \\
\hline $\begin{array}{l}\text { Mean } \\
{[95 \% \mathrm{Cl}]}\end{array}$ & $\begin{array}{l}2.63 \text { [2.52, } \\
2.73]\end{array}$ & $\begin{array}{l}2.633[2.53, \\
2.72]\end{array}$ & $0.99[0.94,1.03]$ & $\begin{array}{l}1.02 \\
{[0.98} \\
1.05]\end{array}$ & $\begin{array}{l}5.06 \text { [4.81, } \\
5.30]\end{array}$ & $\begin{array}{l}4.96[4.83, \\
5.09]\end{array}$ & $\begin{array}{l}1.17 \text { [0.94, } \\
1.39]\end{array}$ & $\begin{array}{l}1.75 \text { [1.60, } \\
1.91]\end{array}$ \\
\hline P-value & 0.97 & & 0.25 & & 0.48 & & $<0.001$ & \\
\hline \multicolumn{9}{|l|}{ Northern } \\
\hline $\begin{array}{l}\text { Mean } \\
{[95 \% \mathrm{Cl}]}\end{array}$ & $\begin{array}{l}6.51 \\
{[6.04} \\
6.98]\end{array}$ & {$[7.11,8.04]$} & $\begin{array}{l}1.53[1.46, \\
1.61]\end{array}$ & $\begin{array}{l}1.80 \\
{[1.73} \\
1.87]\end{array}$ & $\begin{array}{l}4.73 \\
{[4.52} \\
4.95]\end{array}$ & $.81,5.16]$ & $\begin{array}{l}0.37[0.01 \\
0.73]\end{array}$ & $\begin{array}{l}1.20 \text { [0.81, } \\
1.59]\end{array}$ \\
\hline P-value & $<0.01$ & & $<0.001$ & & 0.06 & & $<0.01$ & \\
\hline \multicolumn{9}{|c|}{ Upper East } \\
\hline $\begin{array}{l}\text { Mean } \\
{[95 \% \mathrm{Cl}]}\end{array}$ & $\begin{array}{l}2.79 \text { [2.58, } \\
3.00]\end{array}$ & $\begin{array}{l}2.70[2.56, \\
2.84]\end{array}$ & $\begin{array}{l}0.98 \text { [0.91, } \\
1.05]\end{array}$ & $\begin{array}{l}1.08 \\
{[1.01} \\
1.14]\end{array}$ & $\begin{array}{l}4.73 \\
{[4.53} \\
4.93]\end{array}$ & $.51,4.92]$ & $\begin{array}{l}0.91[0.47, \\
1.35]\end{array}$ & $\begin{array}{l}1.44[1.18, \\
1.70]\end{array}$ \\
\hline P-value & 0.49 & & 0.05 & & 0.90 & & 0.04 & \\
\hline
\end{tabular}




\begin{tabular}{|c|c|c|c|c|c|c|c|c|}
\hline Variables & ANC4 visit & & \multicolumn{2}{|c|}{ Skilled Delivery } & \multicolumn{2}{|c|}{ Maternal Mortality rate } & \multicolumn{2}{|c|}{ Neonatal Mortality Rate } \\
\hline \multicolumn{9}{|c|}{ Upper West } \\
\hline $\begin{array}{l}\text { Mean } \\
{[95 \% \mathrm{Cl}]}\end{array}$ & $\begin{array}{l}3.44[3.12, \\
3.75]\end{array}$ & $\begin{array}{l}4.35[4.04, \\
4.66]\end{array}$ & $\begin{array}{l}1.26[1.18 \\
1.34]\end{array}$ & $\begin{array}{l}1.42 \\
{[1.35,} \\
1.50]\end{array}$ & $\begin{array}{l}4.94 \\
{[4.69,} \\
5.20]\end{array}$ & $4.69[4.50,4.89]$ & $\begin{array}{l}1.32 \text { [1.05, } \\
1.59]\end{array}$ & $\begin{array}{l}1.44[1.18, \\
1.69]\end{array}$ \\
\hline P-value & $<0.001$ & & $<0.01$ & & 0.12 & & 0.53 & \\
\hline
\end{tabular}

ANC four visits saw a statistically significant mean difference in the Central, Western, Ashanti, Northern, Upper West and Brong-Ahafo regions with pre and post-intervention mean estimates of 4.05 (95\% Cl: 3.89, 4.23; p-value< 0.01$)$ and $3.73(95 \% \mathrm{Cl}$ : 3.59, 3.86; p-value< 0.01); 3.31 (95\% Cl: 3.16, 3.45; p-value $=0.04)$ and $3.13(95 \% \mathrm{Cl}: 3.03,3.22 ; \mathrm{p}$-value $=0.04) ; 4.66(95 \% \mathrm{Cl}: 4.40,4.91 ; \mathrm{p}$-value $<0.001)$ and 3.66 (95\% Cl: 3.51, 3.81; p-value< 0.001); 6.51 (95\% Cl: 6.04, 6.98; p-value< 0.01 ) and 7.57 (95\% Cl: 7.11, 8.04; p-value< 0.01$)$; 3.44 (95\% Cl: 3.12, 3.75; $p$-value < 0.001$)$ and 4.35 (95\% Cl: 4.04, 4.66; p-value< 0.001); 2.57 (95\% Cl: 2.39, 2.76; p-value = 0.001$)$ and 2.97 (95\% Cl: 2.83 , $3.11 ; p$-value $=0.001)$ respectively.

The Volta, Western, Brong Ahafo, Northern and Upper West regions recorded a pre and post-intervention mean of 0.93 (95\% Cl: 0.86, 1.01; p-value < 0.001$)$ and 1.09 (95\% Cl: 1.05, 1.13; p-value< 0.001); 1.00 (95\% Cl: 0.96, 1.03; p-value< 0.001$)$ and 1.10 (95\% Cl: 1.06, 1.14; pvalue < 0.001); 2.57 (95\% Cl: 2.39, 2.76; p-value = 0.001) and 2.97 (95\% Cl: 2.83, 3.11; p-value = 0.001); 0.88 (95\% Cl: 0.85, 0.91; p-value< 0.001 ) pre-intervention and 0.98 (95\% Cl: 0.94, 1.02; p-value< 0.001$)$; $1.53(95 \% \mathrm{Cl}: 1.46,1.61 ; \mathrm{p}$-value < 0.001$)$ and 1.80 (95\% Cl: 1.73, 1.83; p-value < 0.001$) ; 1.26(95 \% \mathrm{Cl}: 1.18,1.34$; p-value < 0.01$)$ and $1.42(95 \% \mathrm{Cl}: 1.35,1.50 ; \mathrm{p}$-value< 0.01$)$ respectively for the percentage of women who had skilled deliveries.

Mean maternal mortality rate was statistically significant in the Central $4.57(95 \% \mathrm{Cl}: 4.31,4.82 ; \mathrm{p}$-value $=0.04)$ and $4.87(95 \% \mathrm{Cl}: 4.73$, 5.01; p-value = 0.04), Volta 4.98 (95\% Cl: 4.83, 5.14; p-value = 0.02) and 4.68 (95\% Cl: 4.47, 4.89; p-value = 0.02$)$ and Brong-Ahafo $4.82(95 \%$ Cl: 4.65, 5.00; $p$-value $=0.02)$ and $4.55(95 \% \mathrm{Cl}: 4.39,4.71 ; p$-value $=0.02)$ regions, pre and post-intervention respectively.

Pre and post-intervention mean was significant statistically in the Central region 0.35 (95\% Cl: $-0.12,0.82$; $\mathrm{p}$-value $<0.001)$ and 1.37 (95\% Cl: 1.17, 1.57; p-value < 0.01), Greater Accra region 1.70 (95\% Cl: 1.49, 1.92; p-value< 0.01$)$ and 2.07 (95\% Cl: 1.93, 2.20; p-value< 0.01$)$, Volta region $1.16(95 \% \mathrm{Cl}: 0.99,1.32$; p-value < 0.001$)$ and $1.63(95 \% \mathrm{Cl}: 1.51,1.76 ; \mathrm{p}$-value< 0.001$)$, Ashanti region 0.17 ( $95 \% \mathrm{Cl}:-0.29$, 0.64; p-value) and 1.82 (95\% Cl: 1.45, 2.20; p-value< 0.001), Brong-Ahafo region 1.68 (95\% Cl: 1.54, 1.81; p-value< 0.001$)$ and 1.97 (95\% Cl: 1.89, 2.05; p-value < 0.001), Eastern region 1.17 (95\% Cl: 0.94, 1.39; p-value< 0.001 ) and 1.75 (95\% Cl: 1.60, 1.91; p-value< 0.001$)$, Northern region 0.37 (95\% Cl: 0.01, 0.73; p-value< 0.01 ) and 1.20 (95\% Cl: 0.81, 1.59; p-value< 0.01 ) and Upper East region 0.91 ( $95 \%$ Cl: 0.47, 1.35; $\mathrm{p}$-value $=0.04)$ and $1.44(95 \% \mathrm{Cl}: 1.18,1.70 ; \mathrm{p}$-value $=0.04)$ respectively in the estimated neonatal mortality rates.

\section{Impact of MCHNP on the percentage of women who had four (4) ANC visits using interrupted time series analysis}

Table 4 displays the impact of MCHNP on ANC four visits. The results indicate that five of the ten regions had a significant impact in proportion of women who had four ANC visits following the implementation of the intervention, that is ANC four visits increased but this rise in attendance was not significant statistically. The Central and Western regions of the coastal belt of the country recorded an increase of 0.004 ( $95 \% \mathrm{Cl}:-0.003,0.011$; p-value > 0.05$)$ and 0.003 ( $95 \% \mathrm{Cl}:-0.003,0.008 ; \mathrm{p}$-value $>0.05)$ respectively. The Eastern region was the only region in the middle belt that saw an increase in the porportion of women who received ANC four visit with a post trend change of 0.002 (95\% Cl: -0.004, 0.008; p-value >0.05). Upper East and Upper West regions recorded a positive impact in the percentage of women who received ANC four visit having recorded a post trend change of 0.011 (95\% Cl: $-0.004,0.026$; p-value > 0.05$)$ and 0.007 (95\% Cl: -0.012 , 0.026; $p$-value > 0.05) respectively for the savannah belt as shown in Fig. 1 below. The results presented in the table although indicated a positive impact, none of the regions' impact was said to be statistically significant. 
Table 4

Impact of MCHNP on the percentage of women who had four (4) ANC visits using interrupted time series analysis

\begin{tabular}{|c|c|c|c|c|c|}
\hline \multirow[t]{2}{*}{ Variable } & Central Region & Western Region & Eastern Region & Upper East Region & Upper West Region \\
\hline & $\begin{array}{l}\text { Coefficient }[95 \% \\
\mathrm{Cl}]\end{array}$ & Coefficient [95\% Cl] & Coefficient [95\% Cl] & $\begin{array}{l}\text { Coefficient }[95 \% \\
\mathrm{Cl}]\end{array}$ & Coefficient $[95 \% \mathrm{Cl}]$ \\
\hline Time & $\begin{array}{l}-0.007 * \\
{[-0.01,-0.001]}\end{array}$ & $\begin{array}{l}-0.006 *[-0.010 \\
-0.001]\end{array}$ & $\begin{array}{l}-0.007 * *[-0.012 \\
-0.002]\end{array}$ & $\begin{array}{l}-0.01[-0.023 \\
0.003]\end{array}$ & $\begin{array}{l}-0.005[-0.021 \\
0.010]\end{array}$ \\
\hline Intervention & $\begin{array}{l}0.057[-0.064 \\
0.178]\end{array}$ & $0.076[-0.032,0.185]$ & $0.175^{\star \star}[0.068,0.283]$ & $\begin{array}{l}0.08[-0.188 \\
0.347]\end{array}$ & $\begin{array}{l}0.276[-0.035 \\
0.586]\end{array}$ \\
\hline $\begin{array}{l}\text { Interaction/ } \\
\text { Impact }\end{array}$ & $\begin{array}{l}0.004[-0.003 \\
0.011]\end{array}$ & $0.003[-0.003,0.008]$ & $0.002[-0.004,0.008]$ & $\begin{array}{l}0.011[-0.004 \\
0.026]\end{array}$ & $\begin{array}{l}0.007[-0.012 \\
0.026]\end{array}$ \\
\hline
\end{tabular}

It however appears that ANC four visits decreased in the Greater Accra, Volta, Ashanti, Brong Ahafo and Northern regions as shown in Table 1 of the supplementary material.

\section{Impact of MCHNP on the percentage of women who had skilled delivery using interrupted time series analysis}

Table 5 presents results from the impact analysis for the percentage of women who received skilled deliveries. As presented in the table, six of the ten regions in the country had positive impact on the variable been measured but none of these impacts were statistically significant. Central and Western regions recorded a post trend change of $0.003(95 \% \mathrm{Cl}$ - $-0.006,0.013 ; \mathrm{p}$-value $>0.05)$ and 0.001 (95\% Cl: $-0.006,0.008 ; p$-value $>0.05$ ) respectively in the percentage of women who had skilled delivery. Again the Eastern region recorded an estimated impact of 0.006 ( $95 \% \mathrm{Cl}:-0.002,0.014$; p-value $>0.05)$, as the only region in the middle belt of the country that had a positive impact following the introduction of the intervention as shown in Fig. 2. All the three regions of the savannah belt saw an increase in the post trend change of the percentage of women who had skilled delivery with an estimated impact of $0.002(95 \% \mathrm{Cl}:-0.013,0.018 ; \mathrm{p}$-value $>0.05$ ) for the Northern region, 0.007 (95\% Cl: $-0.007,0.021$; p-value > 0.05) for the Upper East region and 0.005 (95\% Cl: $-0.011,0.021 ; \mathrm{p}-$ value $>0.05$ ) for the Upper West region.

Table 5

Impact of MCHNP on the percentage of women who had skilled delivery using interrupted time series analysis

\begin{tabular}{|c|c|c|c|c|c|c|}
\hline Variable & $\begin{array}{l}\text { Central Region } \\
\text { Coefficient [95\% } \\
\mathrm{Cl}]\end{array}$ & $\begin{array}{l}\text { Western Region } \\
\text { Coefficient } \\
{[95 \% \mathrm{Cl}]}\end{array}$ & $\begin{array}{l}\text { Eastern Region } \\
\text { Coefficient [95\% } \\
\text { Cl] }\end{array}$ & $\begin{array}{l}\text { Northern } \\
\text { Region } \\
\text { Coefficient } \\
{[95 \% \mathrm{Cl}]}\end{array}$ & $\begin{array}{l}\text { Upper East } \\
\text { Region } \\
\text { Coefficient [95\% } \\
\text { Cl] }\end{array}$ & $\begin{array}{l}\text { Upper West } \\
\text { Region } \\
\text { Coefficient } \\
{[95 \% \text { Cl] }}\end{array}$ \\
\hline Time & $\begin{array}{l}-0.003[-0.011 \\
0.006]\end{array}$ & $\begin{array}{l}0.002[-0.005 \\
0.008]\end{array}$ & $\begin{array}{l}-0.005[-0.013 \\
0.002]\end{array}$ & $\begin{array}{l}0.004[-0.009 \\
0.018]\end{array}$ & $\begin{array}{l}0.001[-0.012 \\
0.013]\end{array}$ & $\begin{array}{l}0.001[-0.012 \\
0.014]\end{array}$ \\
\hline Intervention & $\begin{array}{l}0.046[-0.088 \\
0.181]\end{array}$ & $\begin{array}{l}0.039[-0.064 \\
0.141]\end{array}$ & $\begin{array}{l}0.077[-0.023, \\
0.178]\end{array}$ & $\begin{array}{l}0.096[-0.146 \\
0.337]\end{array}$ & $\begin{array}{l}-0.058[-0.306, \\
0.191]\end{array}$ & $\begin{array}{l}0.059[-0.203 \\
0.321]\end{array}$ \\
\hline Interaction/Impact & $\begin{array}{l}0.003[-0.006 \\
0.013]\end{array}$ & $\begin{array}{l}0.001[-0.006 \\
0.008]\end{array}$ & $\begin{array}{l}0.006[-0.002 \\
0.014]\end{array}$ & $\begin{array}{l}0.002[-0.013, \\
0.018]\end{array}$ & $\begin{array}{l}0.007[-0.007 \\
0.021]\end{array}$ & $\begin{array}{l}0.005[-0.011 \\
0.021]\end{array}$ \\
\hline
\end{tabular}

Conversely the Greater Accra, Volta, Ashanti, and Brong Ahafo regions saw a reduced impact in skilled deliveries as shown in Table 2 of the supplementary material.

\section{Impact of MCHNP on Maternal Mortality Rate using interrupted time series analysis}

Following the introduction of the intervention, maternal mortality rates were anticipated to reduce across all the regions of the country. Table 6 displays the post trend change for regions that saw a positive impact in maternal mortality rate, however, these impacts recorded were not statistically signaificant. The Greater Accra, Volta and Western regions of the coastal belt recorded some reduction in maternal 
mortality rates with a post trend change of 0.001 (95\% Cl: $-0.018,0.017$; p-value > 0.05$), 0.010(95 \% \mathrm{Cl}:-0.035,0.016 ; \mathrm{p}$-value > 0.05$)$ and 0.009 (95\% Cl: $-0.031,0.013 ;$ p-value > 0.05) respectively.

Table 6

Impact of MCHNP on Maternal Mortality Rate using interrupted time series analysis

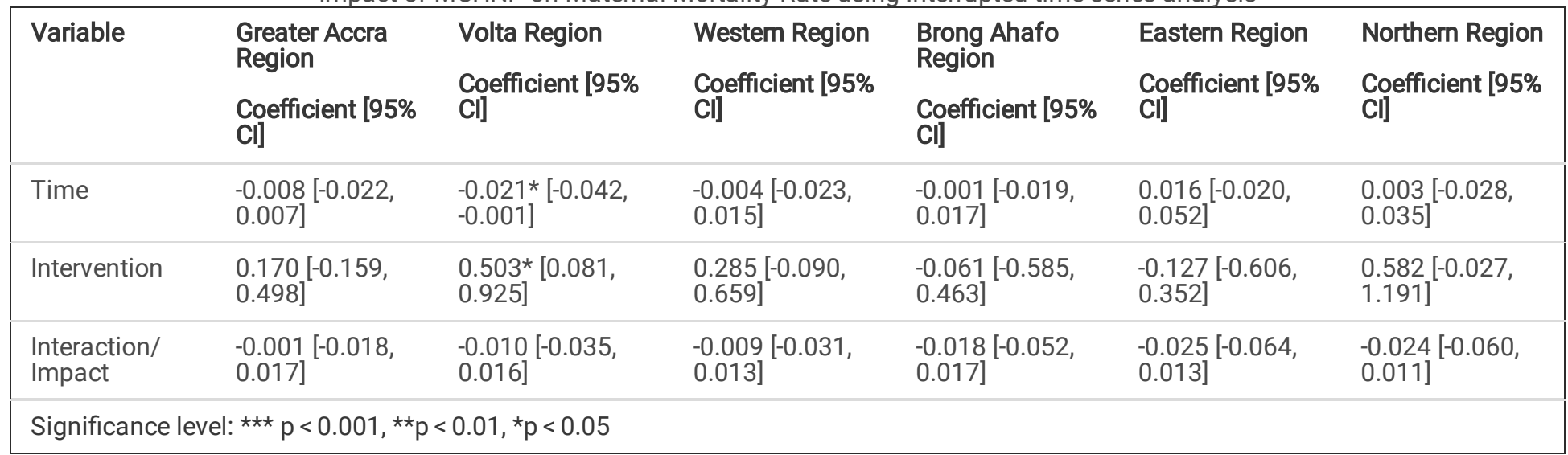

The post trend change for the Brong Ahafo and Eastern regions representing the middle belt was $-0.018(95 \% \mathrm{Cl}-0.052,0.017 ; \mathrm{p}$-value > $0.05)$ and -0.025 (95\% Cl: $-0.064,0.013 ;$-value > 0.05$)$ respectively as shown in Fig. 3.

The Northern region was the only region in the savannah belt that recorded a reduction in maternal mortality rate with a post trend change of 0.024 (95\% Cl: $-0.060,0.011$; p-value > 0.05).

Following the introduction of the intervention, maternal mortality rates, however, saw an increase in the Central, Ashanti, Upper East and Upper West regions shown in Table 3 of the supplementary paper.

\section{Impact of MCHNP on neonatal mortality rate using interrupted time series analysis}

From the ITSA, two regions both in the middle belt of the country saw a fall in the neonatal mortality rate post-intervention. Post trend level change of neonatal mortality rate in the Ashanti region was -0.026 ( $95 \% \mathrm{Cl}:-0.103,0.052$; p-value $>0.05)$ and that of the Brong Ahafo region was lessened by 0.005 ( $95 \% \mathrm{Cl}$ : $-0.028,0.019$; p-value $>0.05$ ) as displayed in Fig. 4. Despite the decline the impact of the outcome in these regions were not statistically significant as presented in Table 7.

Table 7

Impact of MCHNP on Neonatal Mortality Rate using interrupted time series analysis

\begin{tabular}{|lll|}
\hline Variable & $\begin{array}{l}\text { Ashanti Region } \\
\text { Coefficient }[95 \% \mathrm{Cl}]\end{array}$ & $\begin{array}{l}\text { Brong Ahafo Region } \\
\text { Coefficient [95\% Cl] }\end{array}$ \\
\hline Time & $0.064 *[0.004,0.124]$ & $0.008[-0.015,0.030]$ \\
\hline Intervention & $0.191[-1.140,1.522]$ & $0.143[-0.204,0.490]$ \\
\hline Interaction/ Impact & $-0.026[-0.103,0.052]$ & $-0.005[-0.028,0.019]$ \\
\hline Significance level: ${ }^{* \star} \mathrm{p}<0.001,{ }^{* *} \mathrm{p}<0.01,{ }^{*} \mathrm{p}<0.05$ \\
\hline
\end{tabular}

However, it appears that neonatal mortality rate rather increased significantly in the Western, Upper East and Upper West regions ( $p$-value < 0.001). The Central, Greater Accra, Volta, Eastern, and Northern regions also saw an icrease in neonatal mortality rate following MCHNP implementatio as presented in Table 4 of the supplementary material

\section{Discussion}

This paper adds to the other quasi-experimental studies that found mixed impact (see Tables: 4, 5, 6, 7 of the results section and Tables: 1 , 23,4 of supplementary material) of performance-based financing incentives in the arena of health care utilization, health service efficiency and quality as revealed in the result section and supplementary impact analysis of this manuscript. 
Results from the study indicated that some regions recorded positive impact in ANC four visits whereas others did not see the impact as expected. Porportion of women who had four ANC visits in the Central, Western, Eastern, Upper East and Upper West regions had positive impacts. The impacts although in a positive direction, were not statistically significant ( $p$-value $>0.05)$. Conversely the Volta region recorded a statistically significant reduction in the ANC four visit with estimated impact of -0.011 ( $95 \% \mathrm{Cl}:-0.020,-0.002 ; \mathrm{p}$-value $<0.01)$ as shown in supplementary table 1 . The increased attendance are consistent with an impact evaluation of a PBF scheme in the Burkina Faso which asserted that the introduction of performance-based financing (PBF) incentives increased the utilisation and coverage of maternal health services chief of it been increased ANC visits [20].

Early and ongoing monitoring during pregnancy is believed to be associated with favourable birth outcomes. Prenatal care is beneficial in reducing the frequency of preterm deliveries, low birth weight as well as maternal and perinatal mortalities. Despite these effects of having multiple ANC visits, the impact of MCHNP on the recommended four ANC visits seem insignificant in the Greater Accra, Ashanti, BrongAhafo, and Northern regions following its implementation. Although the impact in these regions were in the negative direction it can not be seen as the intervention did not have a positive impact on the variable as the indicator could has worsen should the intervention not been implemented.

Among the factors that could contribute to the insignificant impacts recorded are; poor design of the intervention to suit ones local setting as the one fit all approach does not always work well in many settings. Among the factors are limited availability of resources such as vehicles, logistics and staff, and also the late registration of pregnant women to the clinic later than the WHO recommend first ANC visit of twelve weeks of pregnancy. Again the cost transportation on the clinet to the facility could be a reason to the insignifcant impact.

Following the implementation of MCHNP, uptake of skilled delivery services increased in the Central $(0.003 ; 95 \% \mathrm{Cl}:-0.006,0.013)$, Western (0.001; $95 \%$ Cl: $-0.006,0.008)$, Eastern $(0.006 ; 95 \%$ Cl: $-0.002,0.014)$, Northern $(0.002 ; 95 \% \mathrm{Cl}:-0.013,0.018)$, Upper East (0.011; $95 \% \mathrm{Cl}$ : $-0.004,0.026)$, and Upper West regions $(0.007 ; 95 \% \mathrm{Cl}:-0.012,0.026)$. However none of these positive impacts recorded was significant statistically ( $p$-value >0.05). The increased uptake in the utilization of this service is in congruence with a studies in Bangladesh which asserted that, performance-based incentives increased the number of women who delivered in the health facility by skiiled birth attendants [21]. The study further indicated that the combination of financial incentive packages had a larger effect compared to the only performance incentives [21]. Again results from the study are consistent with the study in Rwanda which reported a significant improvement in institutional deliveries following the implementation of a PBF intervention hence recommended that PBF interventions are the best approaches to improving institutional deliveries in LMICs [22]. It is however indicated that, particular caution be taken when initiating alteration such as the introduction of financial incentive packages in a system as intricate as the health sector to improve coverage and or performance [23].

Post trend analysis revealed a reduction in the percentage of women who had facility deliveries in the Greater Accra, Volta, Ashanti and Brong Ahafo regions. This unexpected impacts could be as a result of unofficial payments, lack of equipment to delivery this essential services in certain health centres, myths surrounding facility deliveries in most of our indigenous settlements, as well as poor collaboration between clients and service providers.

It must however be indicated that, although the impact is in the negative direction for some regions, it does not necessarily equate to the inability of the intervention not producing positive impacts, but rather it must also be seen that the indicator might have seen worst outcomes if the intervention was not implemented.

Greater Accra, Volta, Western, Brong Ahafo, Eastern and Northern regions of Ghana recorded reduction in its maternal mortality rate following the implementation of the performance-based financial incentive, MCHNP. The impact although positive, that is MMR reduced, none of the reduction was significant statistically. Findings of this study are consistent with an impact evaluation study in Rwanda which recorded a reduced maternal mortality rate in the intervention group compared to the control group [22]. According to Africa Progress Panel (2010), performance-based incentives like MCHNP are meant to increase the availability of logistics, provide well-motivated staff to administer essential maternal and child health services to residents in remote settlements to increase coverage and utilisation of available essential care in other to reduce maternal mortalities in the region. The report as stated by Africa Progress Panel also indicated that increased availability and accessibility of essential maternal health and obstetric services to women of reproductive age leads to a massive improvement in curbing the increased rate of maternal mortality.

In contrast, the Central, Ashanti, Upper East and Upper West regions however saw an increase in maternal mortality rates following the implementation of the intervention as shown in Table 3 of the supplementary material. The increased rate might not necessarily be that the intervention did not have a positive impact though the impact is in the the negative direction. This could be that maternal mortality 
rates might have have seen an astronomic increase if the intervention had not been implemented. The unexpected impact of the intervention on this outcome however could be due to reasons elaborated as follows:

Poor design or implementation of the intervention, a one fit all design, that is, adopting broad non-specific ideas to ones' the local setting during the implementation of the financial-based incentive in settings as complex as the health sector. Health system factors such as the poor nature of the Ghanaian referral system from the low facility to the advance facilities for specialised services could be attributed to the unexpected impacts recorded. Again on the health system factors is the inappropriate distribution of critical staff like midwives and obstericains by managers to provide obstetrics and gynaecological and other essential services across the country. Also the lack of consumable and non-conusmable commodities in our facilities such as monitors, foetoscope, ultrasound scans machines (USG), cardiotocography (CTG) machines, blood and blood products and other lifesaving equipment could be linked to the increased maternal mortality rate in the Central, Ashanti, Upper East and Upper West regions.

Following the implementation of the performance-based financing incentive, the Ashanti $(-0.026 ; 95 \%$ Cl: $-0.103,0.052)$ and Brong Ahafo $(-0.00595 \% \mathrm{Cl}:-0.028,0.019)$ regions of the middle belt of the country were the only two regions that saw a positive impact in neonatal mortality rate. The impacts recorded were not significant statistically $(p>0.05)$ despite the regions seeing positive impact as presented in Table 7. Supplementary analysis further indicated a statistically significant increase in neonatal mortality ratein the Western, Upper East and Upper West regions (see Table 4 of supplementary material). The reduction in the rate is in concordance with an evaluation study in Mexico, which reported an increased improvement in child health outcomes following the implementation of PBF where the intervention reduced significantly childhood morbidities and mortalities by declining the occurrence of arrested growth among nursery children indicating an improvement in child outcomes[24, 25]. Conversely, these findings are inconsistent with the impact evaluation conducted in the Philippines following the implementation of PBF which reported that the intervention group of the performance-based incentives reported more morbidities and mortalities than the control group [26].

The Central, Greater Accra, Volta, Eastern, and Northern regions also recorded higher rate of neonatal mortality rates following the implementation of the intervention and this can be seen in Table 4 of the supplementary material of this manuscript. Despite the increased neonatal mortality rates recorded, it does not necessarily mean the intervention did not work in reducing neonatal mortality rate. The intervention might have work even though the impact is in the negative direction as mortality rates might have worsen should the intervention not been implemented.

Poor design of the technical component of the intervention, the use of administrative data, limited skills due to inadequate in-service training for staff in the newborn area on who to help the baby breathe, infection prevention, neonatal resuscitation among others coupled with limited equipment such as incubators, phototherapies, radiant warms among others could be reasons for the insignificant impacts. Among other factors could be the inadequate staff strength in newborn areas, socio-economic and sociodemographic characteristics of parents and the poor referral system.

\section{Strength and Limitation}

The strengths in the application of ITSA are the use of administratively collected data, population been studied acts as its own control, employs modern regression modeling tools and techniques [27]. Among the limitations of the use of ITSA is that, ITSA are sensitive to the choice of the model for evaluating the impact and trend of the intervention and it solely depends on aggregated data for population-level studies as well as diffusion effects in the situation were the lags of events are too long [27].

\section{Conclusion}

The impacts recorded following the implementation of the intervention was statistically significant. ANC four visits increased in the Central, Western, Eastern, Upper East and Upper West regions. Percentage of pregnant women who received skilled deliveries increased in the Central, Western, Eastern, Northern, Upper East and Upper West regions. Greater Accra, Volta, Western, Brong Ahafo, Eastern and Northern regions were the six regions that registered a reduced maternal mortality rate, despite not significnat statistically. Two middle belt regions; Ashanti and Brong-Ahafo were the only two regions that saw a deline in neonatal mortality rate post MCHNP implementation, however the Western, Upper East and Upper regions recorded significant increase in neontatal mortality rate post-intervention.

The study recommends review its technical components and adopt specific designs to address the needs of ones' local setting, to be able to achieve highly significant impacts. 
It is again recommended that further studies including a detailed process evaluation be conducted in regions that the intervention had a strong positive impact to understand why the intervention seems to be trailing in those regions to inform further implementation of similar projects.

\section{Abbreviations}

ANC

Antenatal Clinic

CHIM

Centre for Health Information Management

DHIMS

District Health Information Management System

GHS

Ghana Health Service

IPT

Intermittent Preventive Treatment

ITSA

Interrupted Time Series Analysis

LMICs

Low-Middle Income Countries

$\mathrm{MCH}$

Maternal and Child Health

MCHNP

Maternal and Child Health and Nutrition Improvement Project

MMR

Maternal Mortality Rate

PBF

Performance-Based Financing

PNC

Postnatal Clinic

PPME

Planning, Policy, Monitoring and Evalaution

TBA

Traditional Birth Attendant

\section{Declarations}

\section{Ethical approval and consent to participate}

Permission was sought from Centre for Health Information Management (CHIM) through the Director Planned Policy Monitoring and Evaluation Division (PPM) of the Ghana Health Service (GHS) to use the data from DHIMS2 for this study.

\section{Consent for publication}

Not applicable

\section{Availability of data and material}

The datasets generated and/or analysed during the current study are available in the District Health Information Management System 2 (DHIMS 2) repository; https://dhims.chimgh.org/dhims/

\section{Competing interests}


All authors declared they have no competing interests in carrying out this study.

\section{Funding}

This study received no funding and or grants from anu agency, be it a commercial, non-governemntal or public organization.

\section{Authors' contribution}

AM and JN conceived and designed the study. AM wrote the first draft of the manuscript. DD selected the study design and led the editing and fine-tuning of the manuscript. AM and DD analysed and interpreted the data. JN and DD approved the final manuscript. All authors read and approved the final manuscript.

\section{Acknowledgements}

Authors to this study acknowledge the Ministry of Health and the Ghana Health Service for implementing the intervention and making the data available for use in this study.

\section{References}

1. Kuruvilla S, et al. The Global strategy for women's, children's and adolescents' health (2016-2030): A roadmap based on evidence and country experience. Bull World Health Organ. 2016;94(5):398-400.

2. Report K. "Improving Access to Quality Health and Education Services in the Northern and Upper East Regions of Ghana- An Endline Evaluation: RFP NO: 9127709," 2017.

3. WHO. "WHO policy brief for the Implementation of Intermittent Preventive Treatment of Malaria in Pregnancy Using SulfadoxinePyrimethamine (IPTp-SP)," WHO Press, no. October 2012, pp. 1-13, 2014.

4. Clavagnier I. Antenatal care. " Rev Infirm. 2012;no. 185:45-6.

5. Ganle JK, Kombet ML, Baatiema L. Factors influencing the use of supervised delivery services in Garu-Tempane District, Ghana. BMC Pregnancy Childbirth. 2019;19(1):1-11.

6. Steenland M, et al., "Performance-based financing to increase utilization of maternal health services: Evidence from Burkina Faso," SSM - Popul. Heal., vol. 3, no. July 2016, pp. 179-184, 2017.

7. Ganle JK, Parker M, Fitzpatrick R, Otupiri E. "Inequities in accessibility to and utilisation of maternal health services in Ghana after user-fee exemption: A descriptive study," Int. J. Equity Health, vol. 13, no. 1, 2014.

8. Adu J, Tenkorang E, Banchani E, Allison J, Mulay S. The effects of individual and community-level factors on maternal health outcomes in Ghana. PLoS One. 2018;13(11):1-16.

9. Gabrysch S, Campbell OMR, "Still too far to walk: Literature review of the determinants of delivery service use," BMC Pregnancy Childbirth, vol. 9, no. September 2009, p. 34, 2009.

10. The AIDSTAR-Two P. “The PBF Handbook," in Effective, Implementing Programs, Performance-based Financing, 2011.

11. Management Sciences for Health. "Performance-Based Financing Improves Health Facility Performance and Patient Care in the Democratic Republic of the Congo," no. January, 2017.

12. Sibomana S, Reveillon M, Hera B. "Burundi Case Study: Performance based financing of priority health services," World Heal. Organ., p. 32, 2015.

13. GHS. "Demographic and Health Survey of Ghana," Ghana Stat. Serv., 2014.

14. OMS. "Trends in maternal mortality 1990 to 2015. Estimates developed by WHO, UNICEF, UNFPA and The World Bank.," p. 80, 2015.

15. Paul E, et al. Performance-based financing in low-income and middle-income countries: isn't it time for a rethink? BMJ Glob Heal. 2018;3(1):e000664.

16. Witter S, Fretheim A, Kessy FL, Lindahl AK, "Paying for performance to improve the delivery of health interventions in low- and middleincome countries," Cochrane Database Syst. Rev., vol. 2012, no. 2, 2012.

17. Shadish BWR, Cook TD, Campbell DT, Shadish W, Cook T. "Book Review. 2004;Vol. 27:173-85.

18. Linden A. "A comprehensive set of postestimation measures to enrich interrupted time-series analysis," no. 1, pp. 73-88, 2017. 
19. Mckean J, et al., "Simple Robust Tests for Autocorrelated Errors in Time Series Design Simple Robust Tests for Autocorrelated Errors in Time Series Design Intervention Models," no. June, 2014.

20. Steenland M, et al., "SSM - Population Health Performance-based fi nancing to increase utilization of maternal health services: Evidence from Burkina Faso 区," SSM - Popul. Heal., vol. 3, no. July 2016, pp. 179-184, 2017.

21. Morgan L, et al., “Financial incentives and maternal health: Where do we go from here?," J. Heal. Popul. Nutr., vol. 31, no. 4 SUPPL.2, 2013.

22. Basinga P, Gertler PJ, Binagwaho A, Soucat AL, Sturdy J, Vermeersch CM. Effect on maternal and child health services in Rwanda of payment to primary health-care providers for performance: An impact evaluation. Lancet. 2011;377(9775):1421-8.

23. Adam T, De Savigny D, "Systems thinking for strengthening health systems in LMICs: Need for a paradigm shift," Health Policy Plan., vol. 27, no. SUPPL. 4, pp. 2006-2008, 2012.

24. Rivera JA, Sotres-Alvarez D, Habicht JP, Shamah T, Villalpando S. Impact of the Mexican program for education, health, and nutrition (Progresa) on rates of growth and anemia in infants and young children: A randomized effectiveness study. J Am Med Assoc. 2004;291(21):2563-70.

25. Gertler P. Do conditional cash transfers improve child health? Evidence from PROGRESA's control randomized experiment. Am Econ Rev. 2004;94(2):336-41.

26. Peabody MA, Perryman KL, Hannah M, Smith L, Sanyshyn SM. "Improving Mental Health Outcomes for Young Children Through the Implementation of the Primary Project," vol. 1, no. 1, pp. 40-50, 2017.

27. Gasparrini A, “Interrupted time series Centre for Evaluation \& Centre for Statistical Methodology,” no. May, 2018.

\section{Figures}

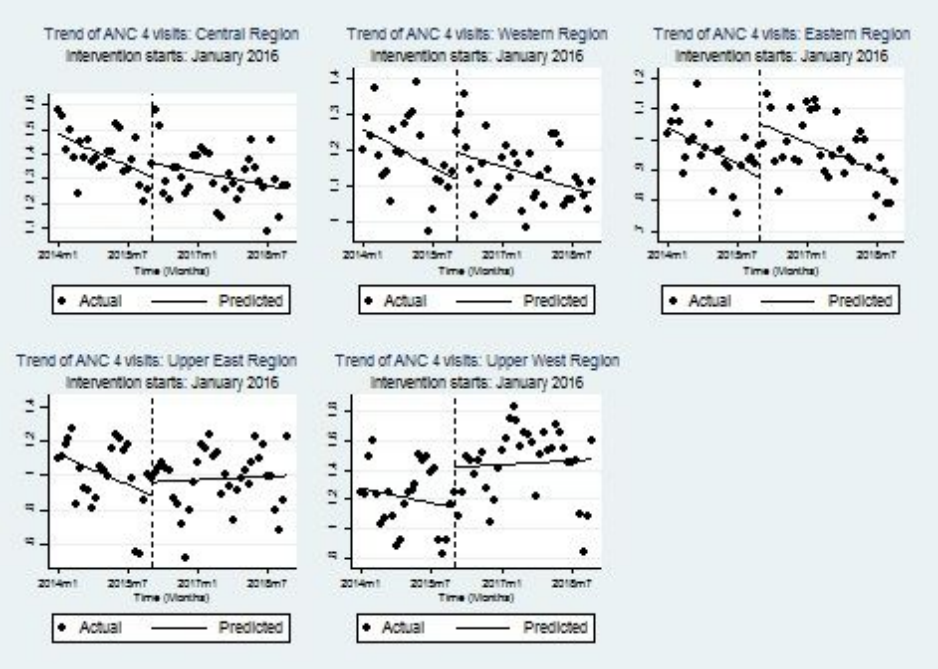

\section{Figure 1}

Trend of ANC four visit in regions that had significant impact 


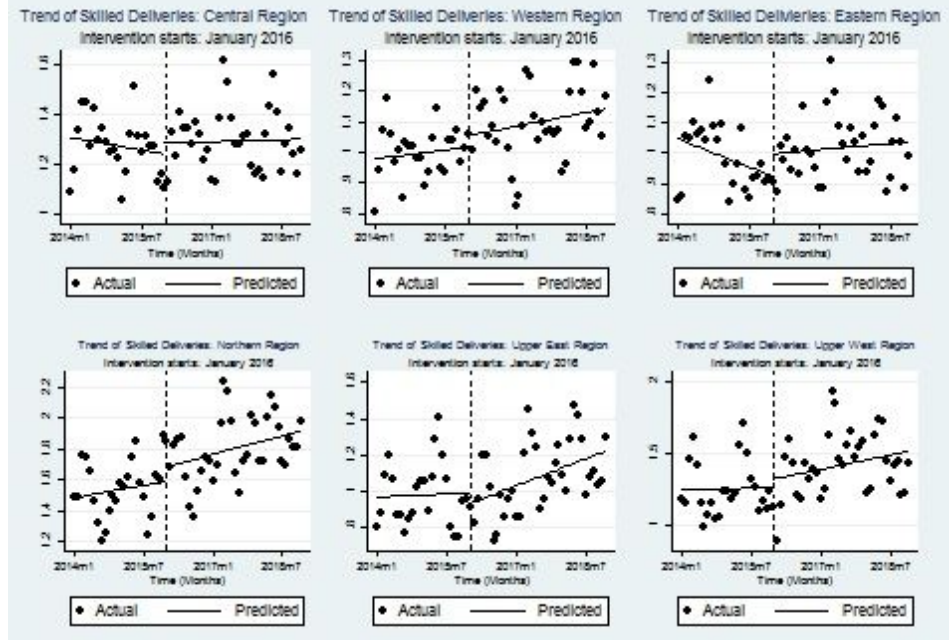

\section{Figure 2}

Trend of skilled delivery in regions that had significant impact

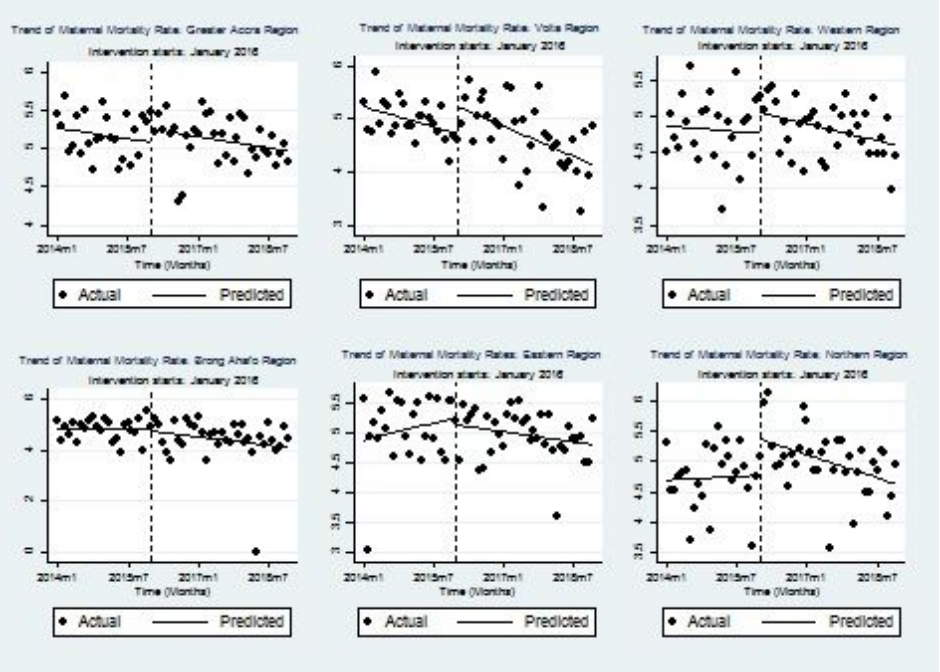

\section{Figure 3}

Trend of maternal mortality rate in regions that had significant impact

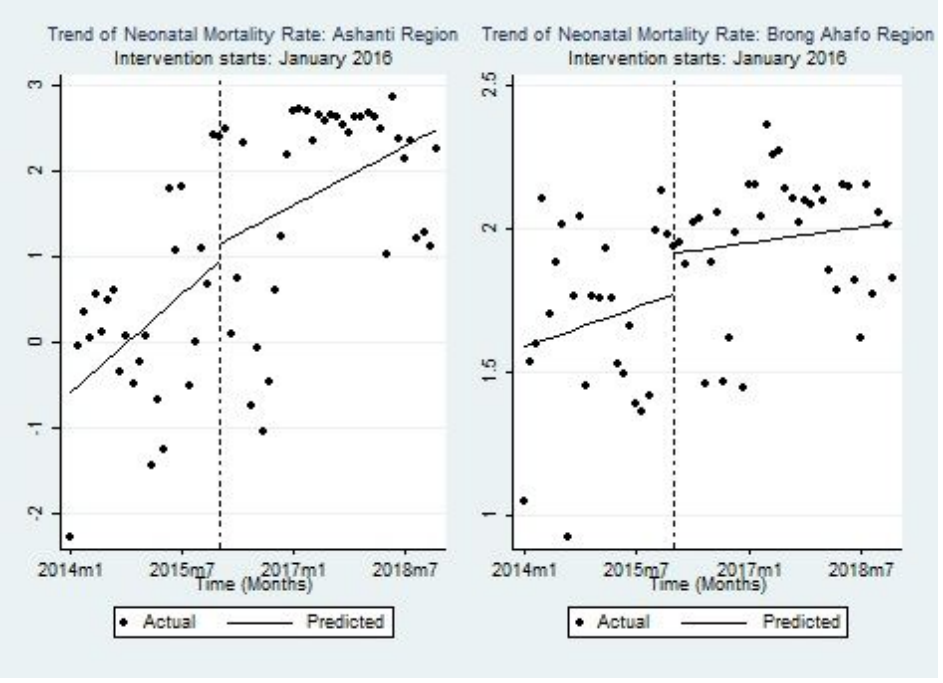

Figure 4 
Trend of neonatal mortality rate in regions that had significant impact

\section{Supplementary Files}

This is a list of supplementary files associated with this preprint. Click to download.

- SupplementaryMaterialMCHNP.docx 